\title{
Technical View of the Super Resolution using Registration, Interpolation and Restoration
}

\author{
Kritika $^{1}$, Vedant Rastogi ${ }^{2}$ \\ M. Tech. Scholar, Department of Computer Science \& Engineering, IET, Alwar, India ${ }^{1}$ \\ Associate Professor, Department of Computer Science \& Engineering, IET, Alwar, India ${ }^{2}$
}

\begin{abstract}
In the paper the most efficient model to implement super resolution using discrete wavelet transform is shown. The model is a three step process of image registration, interpolation and noise filtering using DWT. All the process is explained and simulation result is presented to prove the theory.
\end{abstract}

Keywords: LowResolution, Highresolution, Superresolution, Registration, Interpolation, Restoration, DWT.

\section{INTRODUCTION}

An image sensor or camera is a device which converts optical energy into an electrical signal. Modern imaging sensors are based on charge-coupled-device (CCD) technology which consists of an array of photo detector elements or pixels that have a voltage output proportional to the incident light. The number of detectors elements decides the spatial resolution of the camera. Higher the number of detector elements more is the resolution. A sensor with less number of detector elements produced a low resolution image, giving blocky effect. This is because when a scene is photographed with a resolution camera it is sampled at low spatial sampling frequency causing aliasing effect. One could think of reducing the size of photo detector elements there by increasing the density and hence sampling rate. But if the pixel size decreases the amount of light incident on each pixel also decreases and this causes a shot noise which degrades the image quality. Increasing the pixel density increases the resolution. But also causes shot noise. The current image sensor technology has most reached this level. Another approach to increase the resolution is to increase the wafer sizewhich leads to an increase in the capacitance. This approach is not effective since increases in capacitance causes a decrease in charge transfer rate. This limitation causes the image of a point light source to be blurred. Also there is distortion due to aliasing because of low sampling rate for a low resolution sensor. Moreover in some application like satellite imagery, the physical constraint makes the sensor unrealizable for a high resolution. Thus there is a need for developing post acquisition signal processing technique to enhance the resolution these techniques being post processing methods applied on the low resolution images. They offer flexibility as well as cost benefit. Since there is no additional hardware cost involves. However, the increased computational cost may be the burden that user has to bear.

With the enhancement of the research and the dynamic price change in the image sensor industries now almost everybody is equipped with a camera. Hence the image processing to improve the image quality will directly impacted by this change. Super resolution [1-6] is one of the topics which are still not made to the textbooks on image and video processing. Super resolution refers tothe process of producing a high spatial resolution image from several low resolution images, thereby increasing the maximum spatialfrequency and removing the degradations that arise during the image capturing process using a low resolution camera.

Any given set of source low resolution images only captures a finite amount of information from the scene. The goal of super resolution is to extract the independent information from each image in that set and combine the information into a single high resolution image [10]. The only requirement is that each low resolution image must contain some information which is unique to that image. The non-redundant information from the given low resolution image is used to construct a high resolution image. This means that when these low resolution images mapped on to common reference plane their samples must be sub pixel shifted from samples of other images otherwise the image would contain only redundant information and super resolution reconstruction would not be possible. Most methods in super resolution are reconstruction based that is they are based primarily on uniform and non uniform sampling theorem and do not attempt to create any information not found in low resolution image.

\section{A. SUPER RESOLUTION}

In this paper the approach that is used for super resolution is a three step process i.e. Registration [8] (or Motion estimation), Interpolation and Restoration as seen in the Fig 1. The registration is the process in which out of given low resolution image the reference image is selected and the co-ordinate system of all the other low resolution images is converted in to the reference image co-ordinate system. This is required process because let us assume we have two image but both are not present in the same coordinate system or in other words they are phase shifted to each other that result in the blurring in the image and the SNR of the image will reduce significantly. That means although we will get the high resolution image but that 
image will have less information and more noise. Second step is the interpolation which is a process of generating data points or information from the existing data points or information. So this is main process responsible for the generation high resolution image. And the third and the last improved process are for removing the blurring or any noise from the image using discrete wavelet transform and fusion algorithm.

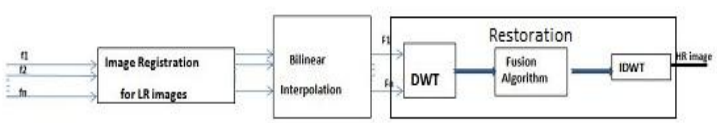

Fig. 1: Improved Super resolution model

\section{REGISTRATION}

Image registration is the process of aligning two or more images of same scene taken at different times, from different viewpoints and/or by different sensors [14]. It is a process of transforming different set of data point into one co-ordinate system. In this paper motion of the image is restricted to shifts and rotation, so a very simple (though accurate) approach is enough for image registration. The following Fig 2 shows different types of registration errors or various forms of degradations.

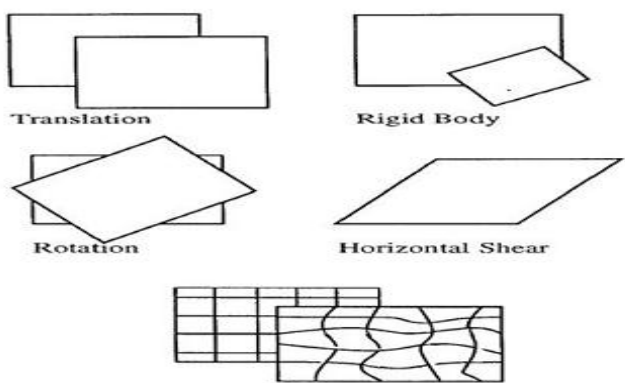

Fig 2: Various types of registration process

Nevertheless, the majority of the registration methods [9] consist of the following four steps.

Feature Detection: Salient and distinctive objects (edges, corners, line intersections, contours, closed-boundary regions) are manually or preferably, automatically detected. For further processing, these features can be represented by their point representatives (line endings, centers of gravity, distinctive points), which are called control points (CPs). Feature Matching: In this step, the correspondence between the features detected in the sensed images and those detected in the reference images is established. Various feature descriptors and similarly measures along with spatial relationship among the feature are used for that purpose.

Transform model estimation: The type and parameters of the so called mapping functions, aligning the sensed image with the reference image, are estimated. The parameters of the mapping functions are computed by means of the established feature correspondence. Image resampling and transformation: The sensed image is transformed by means of mapping functions. Image values in non-integer coordinates are computed by the appropriate interpolation technique.

\section{A.INTERPOLATION ONTO HIGH RESOLUTION GRID}

Once we have a good estimate of shift and rotation between all the images and the reference image, we can find the pixel positions of all the low resolution images in the reference frame of the first image. Then we can project this information on high resolution grid. There are various ways to do this. Here are some most important methods.

Iterative back-projection [6]: This method assumes that each pixel in a low resolution image is result of some integration of pixels in high resolution image that are dependent on camera characteristics. It is very difficult to estimate these projection functions, and so this method tends to be complicated. Also, most of the time camera characteristics are unknown and the parameters have to be tweaked for each set of images.

Papoulis-Gerchberg Algorithm: This method assumes two things:

1. Some of pixel values in the high resolution grid are known.

2. The high frequency components in the high resolution image are zero

3. It works by projecting high resolution grid data on the two sets described above. The steps are:

1. Form a high resolution grid. Set the known pixel values from the low resolution images (after converting their pixel position to the reference frame of first low resolution image). The position on the high resolution grid is calculated by rounding the magnified pixel positions to nearest integer locations.

2. Set the high frequency components to zero in the frequency domain.

3. Force the known pixel values in the spatial domain and iterate.

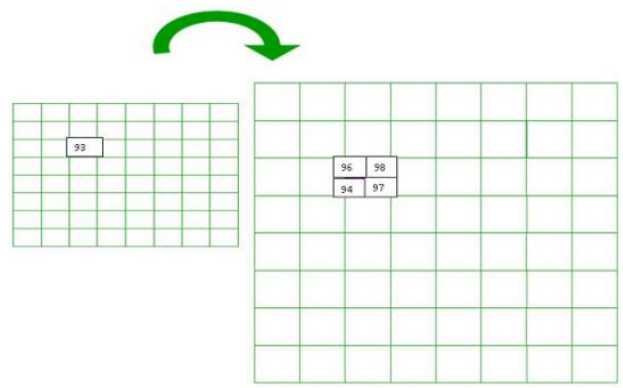

Fig 3: Showing how $i^{\text {th }}$ pixel of Low resolution image is interpolated onto high resolution grid

By making the high frequency equal to zero, this method tries to interpolate the unknown values and so correct the aliasing for low frequency components. Also, by forcing the known values, it does predict some of the high frequency values. The following set of images walks through the actual working of this algorithm. Initially, we fill the high resolution grid with known pixel values and make the unknown pixel values to be zero. The discrete Fourier transform of this initial estimate shows both high and low frequency components. This is about the 
projection on to a high resolution grid and interpolation. In this paper we implemented interpolation techniques like nearest neighbor, bilinear interpolation and cubic interpolations.

\section{B. IMAGE RESTORATION}

The Wavelet series is just a sampled version of continuous wavelet transform and its computation may consume significant amount of time and resources, depending upon the resolution required. The discrete wavelet transform, which is based on sub-band coding is found to yield a fast computation of wavelet transform. In DWT, a time scale representation of the digital signal is obtained using digital filtering techniques. The signal to be analyzed is passed through filters with different cutoff frequencies at different scales.

Filters are one of the most widely used signal processing functions. Wavelets can be realized by iteration of filters with rescaling. The resolution of signal, which is a measure of amount of detail information in the signal, is determined by the filtering operations, and the scale is determined by the amount of up sampling and down sampling operations. The DWT is computed by successive low pass and high pass filtering of the discrete time domain signal as shown in figure 4 . This is called Mall at tree decomposition. Its significance is in the manner it connects the continuous tile multi resolution to discrete time filters. In the figure, the signal is denoted by the sequence $x[n]$, where $n$ is an integer. The low pass filter is denoted by $\mathrm{G}_{0}$ while the high pass filter is denoted by $\mathrm{H}_{0}$. At each level, the high pass filter produces detail information of the signal $\mathrm{d}[\mathrm{n}]$, while the low pass filter with scaling function produces approximations $\mathrm{a}[\mathrm{n}]$.

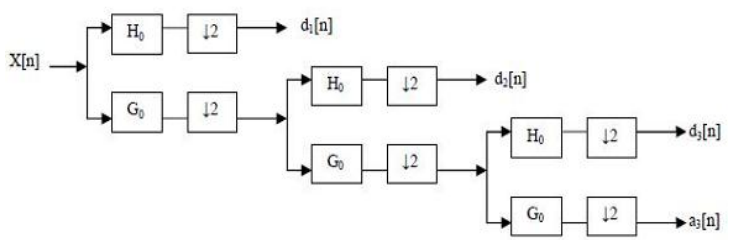

Fig 4: Showing how $\mathrm{i}^{\text {th }}$ pixel of Low resolution image is interpolated onto high resolution grid

So now according to the modified super resolution diagram, firstly LR images is registered. After registration all the images are interpolated using bilinear interpolation. After that we have $\mathrm{k}$ frames and these are applied to the wavelet transform. Usually in all wavelets methods the fusion process is done by taking either maximum or minimum value of the wavelet coefficients and then fuse. Here we fused all the in a meaning full manner. The wavelet coefficients are fused based on the regional variance algorithm. The fluctuation or divergence in the wavelet coefficients is described by the variance in selected region. The covariance of the corresponding region describes the differences between two images. And the correlation coefficient comprised of the mean value, variance and covariance describes the relativity of two images. Considering the smooth coefficients keep the overall image information very well, its difference is far from the detail coefficients. The smooth coefficients are fused by taking the maximum value of the approximate wavelet coefficient. As to the detail coefficients (High frequency coefficients), we take steps as follow

1. The mean $m$, variance $\sigma^{2}$ and the covariance $\beta^{2}$ of the wavelet coefficients are calculated in the certain window $(5 \times 5)$.

2. The correlation coefficients and the weighing coefficients is calculated and then we have to fuse.

3. The correlation coefficient is $\gamma=\beta^{2} / \sigma_{1} \sigma_{2}$, where $\sigma_{1}$ and $\sigma_{2}$ is standard deviation of the corresponding region in two images. The weighing coefficient is $\operatorname{kmax}=1-\gamma / 2$ and $\mathrm{kmin}=\gamma / 2$. And the fused wavelet coefficients are shown in equation

$f_{3}(i, j)=k \max * \max \left\{f_{l}(i, j), f_{2}(I, j)\right\}+k \min * \min \left\{f_{l}(i\right.$, $\left.j), f_{2}(I, j)\right\}$

Finally the reconstructed image is obtained after applying inverse discrete wavelet transform (IDWT).

\section{SIMULATION RESULTS}

Computer simulations are performed to verify the discussed super resolution technique using MATLAB. Set of images from Google earth as shown in Fig 5 is taken which are little bit rotated to each other is used as test image to test the registration process. So first image will act as reference image and we will convert the second image in to the reference co-ordinate system.
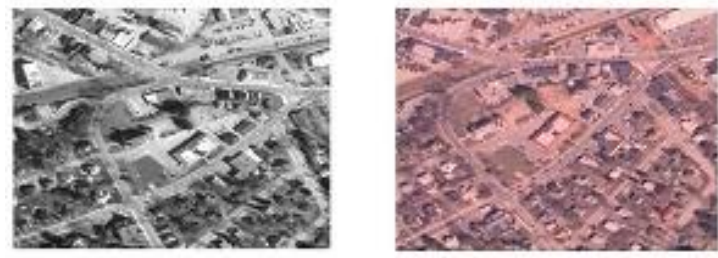

Fig 5: Original Image

So now for second image we applied Projective Transformation by selecting the control points (which should be common in both the images) and shift the required image to the reference image. The result is as seen in Fig 6.
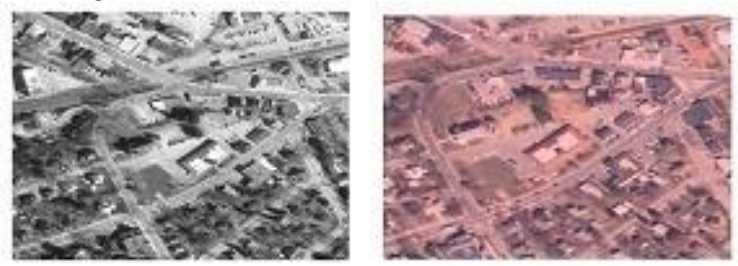

Fig 6: After Registration

The set of low resolution blurred images which are used as the test dataset for simulating the basic approach model of super resolution is seen in Fig 7 .

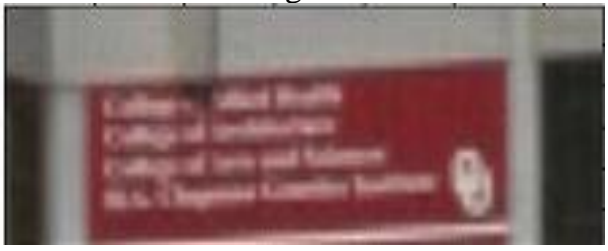

Fig 7: Low resolution image for super resolution model testing 
The image which is obtained after interpolation of the basic super resolution model is seen in Fig 8.

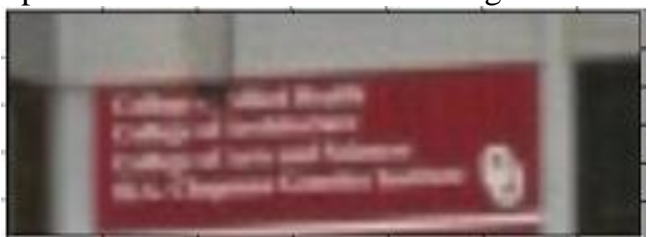

Fig 8: Interpolated image

The final super resolution image which is obtain after applying the restoration stage using discrete wavelet transform and fusion algorithm can be seen in the Fig 9.

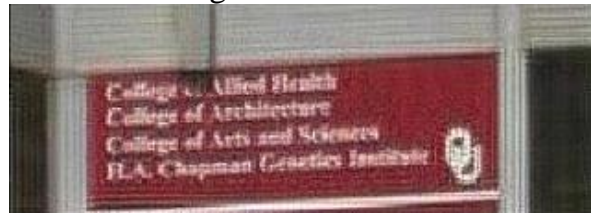

Fig 9: Final High Resolution Image

\section{CONCLUSION}

In the paper a improved super resolution model is proposed by modifying the restoration stage. All the stage is discussed properly with improved restoration stage. The simulation result is performed to check the theory and can be seen that the final image is an improved result. And there is lot of improvement in the super resolution model to improve the further quality of image.

\section{REFERENCES}

[1]. R.Y. Tsai ,T.S. Huang, "Multi-frame image restoration and registration", Advances in computer vision and image processing, Vol. 1(2), 1984, pp.317-339

[2]. S.C. Park, M.K. Park and M.G. Kang "Super Resolution Image Reconstruction: A Technical Overview", IEEE Signal Processing Magazine 3(20),21-36(2003)

[3]. T.Komatsu, K.Aizawa, T. Igarashi, and T. Saito, "Signal processing based method for acquiring very high resolution image with multiple cameras and its theoretical analysis," Proc. Inst. Elec. Eng., vol. 140, no.1, pt. I,pp. 19-25, Feb.1993.

[4]. S. Borman and R.L. Stevenson, "Spatial resolution enhancement of low-resolution image sequences. A comprehensive review with directions for future research," Lab. Image and Signal Analysis, University of Notre Dame,Tech.Rep.,1998

[5]. S. Borman and R.L. Stevenson, "Super-resolution from image sequences - A review," in Proc. 1998 Midwest Symp. Circuits and Systems, 1999, pp. 374 - 378.

[6]. S. Chaudhuri, Ed., Super-Resolution Imaging. Norwell,MA :Kluwer,2001.

[7]. H. Ur and D. Gross, "Improved resolution from sub-pixel shifted pictures, "CVGIP: Graphical Models and Image Processing, vol. 54, pp. 181-186, Mar.1992.

[8]. T. Komatsu, T. Igarashi, K. Aizawa, and T. Saito, "Very high resolution imaging scheme with multiple different-aperture cameras," Sinal Processing: Image Commun., vol. 5, pp. 511-526, Dec. 1993.

[9]. M.S. Alam, J.G. Bognar, R.C. Hardie, and B.J. Yasuda,"Infrared image registration and high-resolution reconstruction using multiple translation ally shifted aliased video frames," IEEE Trans. Instrum. Meas.,vol.49,pp.915-923,Oct.2000.

[10]. N.R. Shah and A. Zakhor, "Resolution enhancement of color video sequences," IEEE Trans. Image Processing, vol. 8, pp. 879-885, June 1999.

[11]. N. Nguyen and P. Milanfar "An efficient wavelet-based algorithm for image super resolution," in Proc. Int. Conf. Image Processing, vol. 2, 2000, pp.\351-354. 